\title{
NONINVASIVE DETECTION OF Cardiovascular Pulsations by Optical DOPPLER TECHNIQUES
}

\author{
HyunDae Hong ${ }^{\dagger}$ and Martin Fox \\ †Akron City Hospital, Biostereometrics Lab/MED-2, 525 E. Market St., Akron, Ohio 44309; \\ †University of Connecticut, Dept. ESE, Central Laboratory for Imaging Research, U-157, \\ 260 Glenbrook Road, Storrs, Connecticut 06269-3157 \\ (Paper JBO-109 received Aug. 26, 1996; revised manuscript received July 7, 1997; accepted for publication July 29, 1997.)
}

\begin{abstract}
A system has been developed based on the measurement of skin surface vibration that can be used to detect the underlying vascular wall motion of superficial arteries and the chest wall. Data obtained from tissue phantoms suggested that the detected signals were related to intravascular pressure, an important clinical and physiological parameter. Unlike the conventional optical Doppler techniques that have been used to measure blood perfusion in skin layers and blood flow within superficial arteries, the present system was optimized to pick up skin vibrations. An optical interferometer with a 633-nm He:Ne laser was utilized to detect micrometer displacements of the skin surface. Motion velocity profiles of the skin surface near each superficial artery and auscultation points on a chest for the two heart valve sounds exhibited distinctive profiles. The theoretical and experimental results demonstrated that the system detected the velocity of skin movement, which is related to the time derivative of the pressure. The system also reduces the loading effect on the pulsation signals and heart sounds produced by the conventional piezoelectric vibration sensors. The system's sensitivity, which could be optimized further, was $366.2 \mu \mathrm{m} / \mathrm{s}$ for the present research. Overall, optical cardiovascular vibrometry has the potential to become a simple noninvasive approach to cardiovascular screening. () 1997 Society of Photo-Optical Instrumentation Engineers. [S1083-3668(97)00604-7]
\end{abstract}

Keywords Doppler interferometry; Michelson interferometer; blood pressure; skin surface displacement.

\section{INTRODUCTION}

Along with blood pressure, the arterial pulse has long been known as one of the most important medical signs and an important indicator of cardiovascular disease. Clinically useful parameters that can be obtained directly or indirectly from the pulsation profile include shape, rhythm, and amplitude of pulsation peaks. ${ }^{1}$ Any change in diameter of an artery directly affects both pressure and flow wave forms. Narrowing of the vascular lumen (inner space), referred to as stenosis, alters the vascular impedance and in turn modifies the contour of the pressure wave and the pulsation or surface deformation profile. ${ }^{2}$

Conventional noninvasive techniques to detect cardiovascular pulsation, such as stethoscopes, phonocardiograms, or manual palpation of superficial arteries, are limited in accuracy, sensitivity, documentation, detected frequency range, and user and patient friendliness. Laser Doppler for cardiovascular applications was introduced in late 1970s. ${ }^{3}$ An optical Doppler shift can be produced through interaction between incident photons and red blood

Address all correspondence to HyunDae Hong. Tel: (330) 375-3561; Fax: (330) 375-3828; E-mail: hhong@brain.biomed.uakron.edu cells. $^{4,5}$ Therefore the frequency spectrum of optical Doppler signals can vary with the rate of blood flow. ${ }^{5}$ In detecting blood flow-related signals, the principle involved in conventional optical Doppler techniques is similar to that of continuous wave (CW) ultrasound Doppler. The Doppler shift of a laser beam was utilized by several researchers to measure local microcirculation. ${ }^{6,7}$ Tissue perfusion imaging was accomplished by scanning a tissue surface with a laser beam and measuring the Doppler-shifted component of backscattered light. ${ }^{8}$

Unlike previous studies, the present work was directed toward detection of skin pulsation. Pulsatile movement of an artery (arterial wall) results in movement of the overlying skin surface. ${ }^{9}$ Near a stenotic lesion in an embedded vessel, the maximum gradient of skin displacement has been shown to be quite distinctive. ${ }^{9}$ In addition, such surface deformations are closely related to pressure. A holographic interferometer was used to map skin surface motion, which demonstrated the feasibility of detecting pulsatile motion of a vessel wall at the skin surface by optical interferometry. The optical interferometer we developed was designed to specifically measure skin vibration profiles related to

1083-3668/97/\$10.00 @ 1997 SPIE 


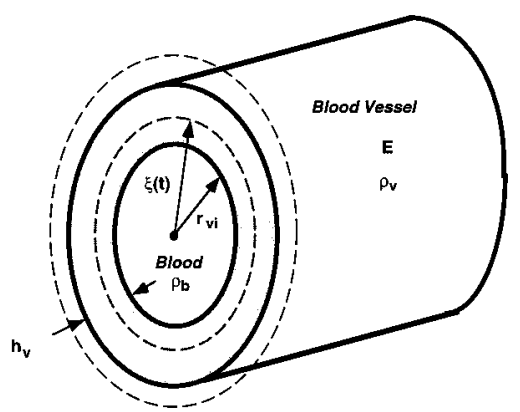

Fig. 1 Model for blood vessel and phantom tube. The two dashed circles indicate the instantaneous inner (intimal) and outer (adventitial) radii of the vessel wall. Variations in these radii are caused by systolic blood pressure. The variables are those given in the text: $h_{v}$ for wall thickness, $\xi(t)$ for an instantaneous luminal radius, $r_{v i}$ for a diastolic luminal radius, $\rho_{b}$ for density of blood, $E$ for an elastic modulus, and $\rho_{v}$ for density of the vessel wall.

the underlying vasculature and so to the pulsation.

\section{MATERIALS AND METHODS}

The blood vessel model for analysis is shown in Figure 1. The time-varying radial dilation of the vessel wall $\xi(t)$ at a fundamental angular frequency $\omega_{0}$ of a pressure wave with a peak magnitude $P_{o}$, can be expressed as ${ }^{10,11}$

$$
\xi(t)=\operatorname{Re}\left[\frac{3}{8} r_{v i} \frac{P_{o} e^{j \omega_{o} t}}{\rho_{v} c_{o}^{2}}\right],
$$

which is realistic for major arteries embedded in tissue layers. The $r_{v i}$ and $\rho_{v}$ are the internal radius under no strain and the density of the blood vessel wall, respectively. $c_{0}$ is the true blood pressure wave velocity and is equal to $\left[E h_{v}^{2} /\left(2 r_{v i} \rho_{b}\right)\right]^{1 / 2}$ where $E, h_{v}$, and $\rho_{b}$ are the elastic modulus, the thickness of the vessel wall, and the density of blood, respectively. The exact value of the Poisson ratio $(\sigma)$ for typical canine arteries is 0.49977 because the bulk modulus of the arterial wall is close to that of water, $2.2 \times 10^{9}\left(\mathrm{dyn} / \mathrm{cm}^{2}\right) .^{2}$ The dilation velocity of a vessel wall $v_{v}$ when the pressure wave form consists of $N$ harmonics, can be obtained from the real part of the time derivative of Eq. $(1)^{12}$ :

$$
v_{v}=\sum_{n=1}^{N} \frac{3}{4} \frac{\rho_{b}}{\rho_{v}} \frac{r_{v i}^{2}}{E h_{v}^{2}} K_{n} P_{o} \sin \left(n \omega_{o} t\right),
$$

where $K_{n}$ is the coefficient of the $n$th harmonic after differentiation of the pressure profile with respect to time. The radial velocity of vessel wall dilation $v_{v}$ can be empirically estimated as ${ }^{13,14}$

$$
v_{v} \approx r_{v i} C_{\alpha} \frac{d P_{t m}}{d t}
$$

where $C_{\alpha}$ is a first-order compliance factor of the vessel wall with transmural pressure $P_{t m}$.
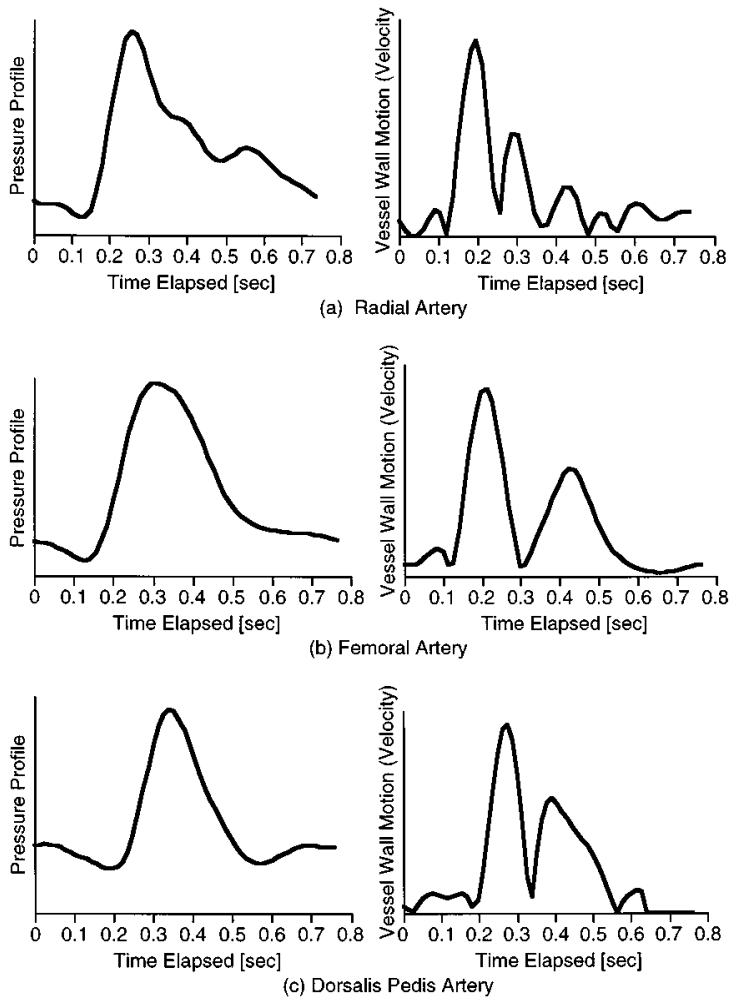

Fig. 2 Theoretical dilation velocity of a vessel wall. Pressure waves are on the left and the corresponding arterial wall motions are on the right [Eq. (2)] at the radial (a), femoral (b), and dorsalis pedis (c) arteries. The pressure wave profiles follow the experimental data presented by O'Rourke, Kelly, and Avelio. ${ }^{14}$ The following values for the parameters of Eq. (2) were used to obtain the wave forms on the right; $r_{v i}=0.174 \mathrm{~cm}$ for $(a), 0.216 \mathrm{~cm}$ for (b), and $0.13 \mathrm{~cm}$ for $(\mathrm{c}) ; \eta_{b}$ (viscosity of blood) $=4.0$ poise, $\rho_{b}$ (density of blood) $=1.055 \mathrm{~g} / \mathrm{cm}^{3}$, and $P_{\text {o }}$ (peak magnitude) $=120$ $\mathrm{mmHg}$.

From Eq. (3), the expected vessel wall dilation velocity for three typical peripheral arteries-the radial, femoral, and dorsalis pedis-can be calculated and is shown in Figure 2. For convenience, only one period of the cardiac cycle is shown. The blood pressure profiles shown at the left in the figures were obtained from the experimental data of O'Rourke, Kelly, and Avolio. ${ }^{14}$ Only the magnitude of velocity is given because the developed system does not independently determine the direction of skin surface motion. The motion profile of a vessel wall for each artery is distinctive due to the pressure profile; the shape and duration of the primary and subsequent peaks in the optical signal profile can be compared with the experimentally obtained profiles shown in the results section. The differences between the theoretical (Figure 2) and empirical (Figures 9 and 10) profiles might be due to the different blood pressure profiles, which vary according to gender, age, vascular elasticity, and the mechanical property of overlying skin layers.

A phantom simulating the viscoelastic properties of a vessel wall (see Figure 3) was fabricated. A latex tube is known to be elastic, so that thickness 


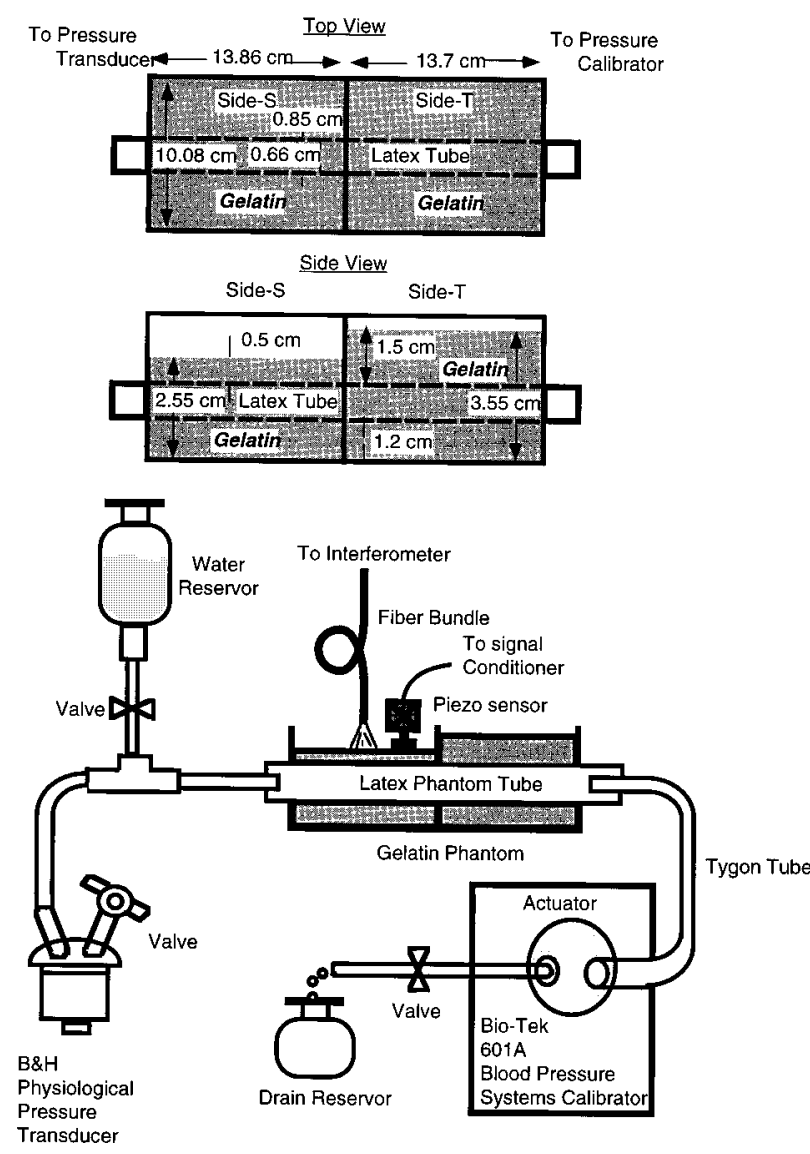

Fig. 3 Gelatin layers and an elastic tube phantom. At the top of the figure, the design of the gelatin phantom is shown with the corresponding dimensions. The latex tube imbedded in gelatin layers is shown as dashed lines. The schematic at the bottom shows how the gelatin phantom and the pressure wave generator were connected in the experimental arrangement. The latex tube has inner and outer diameters of 0.66 and $0.85 \mathrm{~cm}$, respectively.

and internal radius change according to the difference between internal and external pressures. ${ }^{15}$ Like blood vessels, small deformations of an elastic tube are almost linear; the internal radius of the tube varies linearly in response to pressure variations, while large deformations cause nonlinear radial variations. ${ }^{16,17}$

A latex phantom $(0.66-\mathrm{cm}$ internal diameter and 0.095-cm thickness) within a gelatin block was connected to a pressure transducer and a blood pressure calibrator (Figure 3). Gelatin with water (80 to $90 \%$ ) was used to simulate overlying skin tissues. ${ }^{18}$ There are two overlying gelatin layers with different thicknesses; 0.61 (side-S) and $1.2 \mathrm{~cm}$ (side-T) from the bottom. Therefore the effect of the overlying gelatin layer, which simulated skin layers, could be tested. The partition wall in the middle was Plexiglas.

A programmed pressure wave from a pressure calibrator (Blood Pressure Systems Calibrator, BioTek, Model 601A) was used to apply transmural pressure on the phantom vessel and surrounding

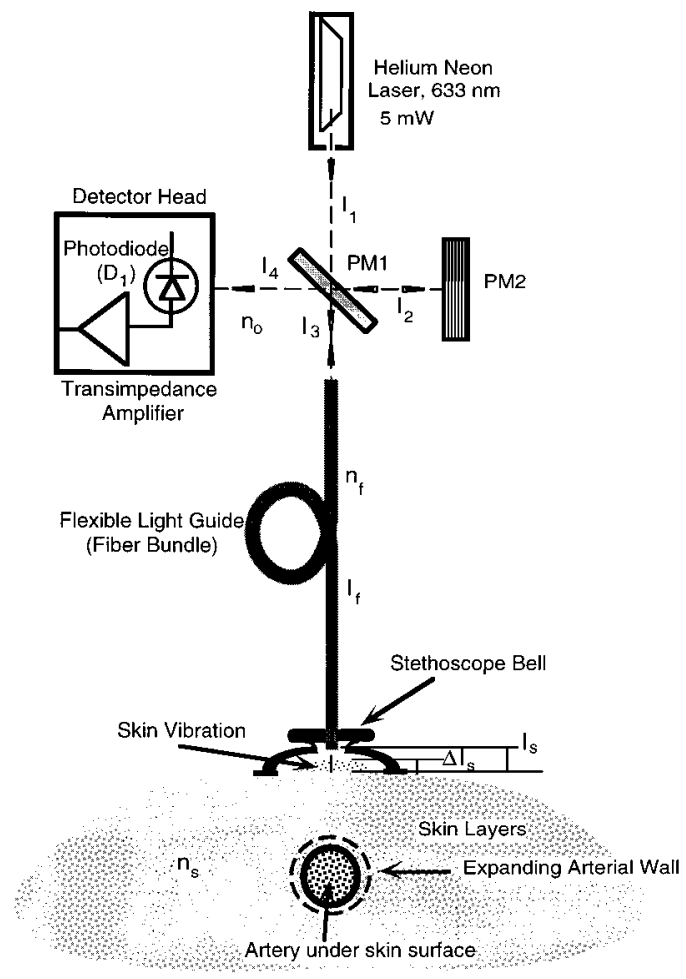

Fig. 4 Optical interferometry. A partial mirror (PMI) and plane mirror (PM2) are mounted on a single optical plate. Optical paths $I_{1}, I_{2}, I_{3}$, and $I_{4}$ are distances from the geometrical center of PM1. The photodetector was installed within an electromagnetically shielded case to minimize noise effects on the detected Doppler signal. $n_{0}, n_{f}$, and $n_{s}$ are indices of refraction for air, fiber bundle (equivalent), and skin layers (equivalent). $I_{s}$ and $\Delta I_{s}$ are the static and the net dynamic distances from an exit pupil of the fiber to the skin surface.

gelatin layer. A piezoelectric transducer was used to detect the motion of the gelatin surface. The tip of the piezosensor gently contacted the surface of the phantom at a position near the light spot of the apical system. The piezoelectric element generated a current signal that was proportional to the time derivative of the pressure. ${ }^{13}$

The basic optical arrangement, shown in Figure 4, followed a Michelson interferometer approach. The He:Ne laser (NEC GL, G5269) emitted approximately $5 \mathrm{~mW}$ of power at $633 \mathrm{~nm}$. The partial mirror had about $50 \%$ power transmission. The initial ray was split into reference and probing rays so that the reference ray followed the path, $l_{1} \rightarrow l_{2} \rightarrow l_{4}$, and the probing ray followed the path, $l_{1} \rightarrow l_{3} \rightarrow l_{f} \rightarrow l_{s}$ $\left(+\Delta l_{s}\right) \rightarrow l_{s}\left(+\Delta l_{s}\right) \rightarrow l_{f} \rightarrow l_{3} \rightarrow l_{4}$ where $\Delta l_{s}$ is the vibration depth. The electric field $E_{s}$ of the laser light can be represented as

$$
E_{s}=E_{0} \cos \left(2 \pi f_{0} t\right)=E_{0} \cos \left(\frac{2 \pi c}{\lambda} t\right),
$$

where $E_{0}$ is the amplitude of the electric field, $c$ is the speed of light in air $\left(3.01 \times 10^{8} \mathrm{~m} / \mathrm{s}\right)$, and $\lambda$ is the wavelength of a laser. Because of the path 


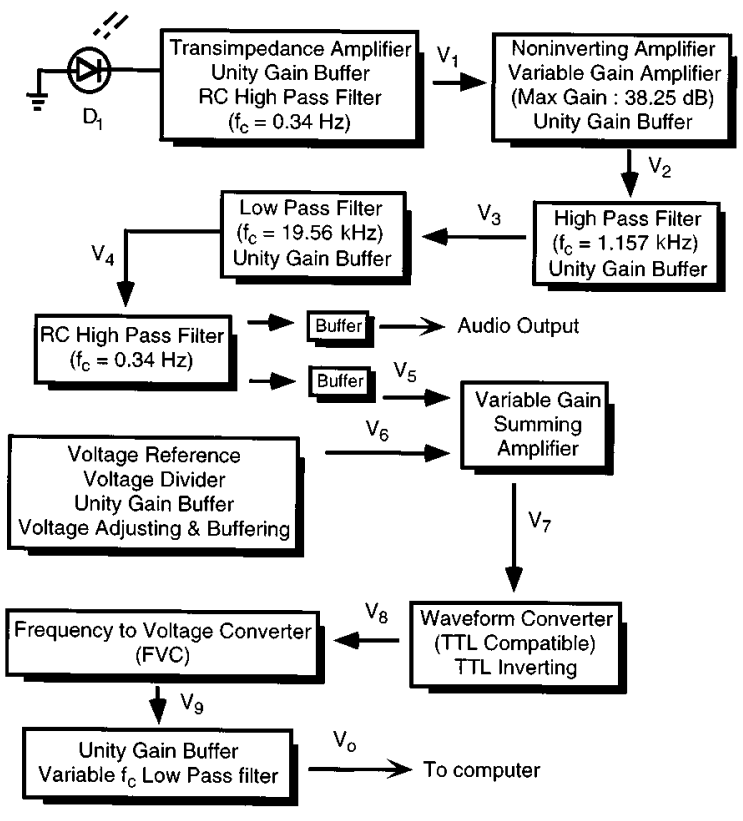

Fig. 5 Block diagram of the instrumentation. The cathode of a photodiode $\left(D_{1}\right)$ is connected to a transimpedance amplifier by an external coaxial cable. Output voltages or signals of each functional block are denoted by $V_{1}$ through $V_{8}$.

length difference due to the skin's surface motion, a time-varying frequency signal $\omega_{D}$ was detected. It can be expressed as

$$
\omega_{D}=2 \pi f_{D}=\frac{d}{d t}\left[\theta_{c}+2 \pi\left(2 \frac{\Delta l_{s}}{\lambda}\right)\right]=\left(\frac{2 \pi}{\lambda}\right) 2 \frac{d}{d t} \Delta l_{s},
$$

where the $\theta_{c}$ is a constant-phase term due to the optical path length difference, which originates from the path geometry of the reference and probing rays. Therefore, when the skin surface is optically homogeneous, the Doppler frequency of a detected signal $f_{D}$, is given as

$$
f_{D}=2 \frac{v_{s}}{\lambda}
$$

where the skin vibration velocity $v_{s}$ is defined as $d \Delta l_{s} / d t$.

As shown in Figure 4, the probing ray was combined with the reference ray on the surface of a photodiode (Hamamatsu, silicon photodiode S1226-5BQ). The laser beam was guided to the skin surface by a flexible fiber optic bundle (Edmund Scientific Co., G40640, 786 fibers). The total length of the fiber bundle was $60 \mathrm{~cm}$.

The electronic processing of the photodiode signal can be understood by referring to Figure 5. All operational amplifiers used in the system had a sufficiently large bandwidth $(20 \mathrm{kHz})$ for the detected Doppler frequencies. The dc level of the signal input to the wave form converter was adjusted by a variable gain summing amplifier. Such adjustment of the dc threshold level was useful in reducing false zero crossings caused by analog noise in a fashion similar to that in ultrasound Doppler units. A stable $10-\mathrm{V}$ dc signal $V_{6}$ was added to the Doppler signal $V_{5}$. The output signal of the wave form converter varied whenever the input signal $V_{5}$ exceeded this threshold level. Therefore the frequency and duty cycle of the output pulse train of the wave form converter were linearly related to the amplified and filtered photodiode signal $V_{5}$.

The frequency-to-voltage converter takes the pulse train coming out of the wave form converter and produces a signal $V_{9}$, which is a summation of triangularlike pulses whose magnitude depends on the period of the Doppler signal $\left[T_{D}=1 / f_{D}\right.$; Eq. (6)]. The final low-pass filter eliminates any ripples at output of the frequency-to-voltage converter.

A 633-nm He:Ne laser beam can penetrate up to $600 \mu \mathrm{m}$ into skin layers. ${ }^{19}$ Therefore, photon scattering within skin layers can modify the diffusely reflecting beam at the skin surface, either as a function of power or beam angle. ${ }^{13}$ Experiments were performed to clarify the effect of photon scattering within skin layers on the detected optical signals.

Laser beam penetration into skin layers was blocked in three ways: (1) by optically opaque polystyrene film, (2) by opaque polystyrene film with white paint on one side of the film, and (3) by direct white paint on the skin surface. The opaque film without painting attenuated incident laser power by $4.93 \mathrm{~dB}$. In the case of opaque film with white painting, optical attenuation was $32.2 \mathrm{~dB}$. Optical power was measured with an optical power meter (Model 401B, 632.8 nm, Spectra Physics Co.).

Table 1 summarizes the human subjects who volunteered for experiments. Institutional Review Board approval was obtained prior to experimentation for clarification of laser safety and the rationale for using human subjects. All subjects voluntarily provided informed consent. We attempted to achieve diversity in age groups, race, gender, and health status in the subject population. Skin characteristics and laser light reflectivity are known to vary according to age and melanin concentration of the skin. ${ }^{19}$

Data were taken at eight different sites for manual palpation of rhythm and strength of arterial pulse; radial, brachial, carotid, temporal, popliteal, femoral, posterior tibial, and dorsalis pedis. Three cardiac auscultation points were assessed by stethoscope: pulmonary (second left intercostal), tricuspid (lower left sternal border), and mitral valves (fifth intercostal space at the midclavicular line).

\section{RESULTS}

To calibrate the entire system, a simulated output signal of the photodiode with transimpedance amplifier was supplied to an input of the unity gain buffer (see Figure 5). The system output, $V_{0}$, in dc millivolts and frequency of Doppler signal $f_{\text {in }}$ in 
Table 1 Specifications of human subjects.

\begin{tabular}{|c|c|c|c|c|c|}
\hline Subject ID & Age & Sex & $\begin{array}{l}\text { Blood } \\
\text { pressure }\end{array}$ & Race & $\begin{array}{l}\text { Health } \\
\text { status }\end{array}$ \\
\hline HUM-01 & 17 & $M$ & $120 / 90$ & Mongolian & \\
\hline HUM-02 & 32 & $M$ & $120 / 80$ & Mongolian & \\
\hline HUM-03 & 16 & $\mathrm{~F}$ & $110 / 80$ & Indian & \\
\hline HUM-04 & 36 & $M$ & $130 / 100$ & Mongolian & \\
\hline HUM-05 & 74 & $M$ & $142 / 85$ & Caucasian & $\begin{array}{l}\text { Pacemaker } \\
\text { implanted }\end{array}$ \\
\hline HUM-06 & 20 & $\mathrm{~F}$ & $120 / 90$ & Indian & \\
\hline HUM-07 & 47 & $M$ & $120 / 80$ & Caucasian & \\
\hline HUM-08 & 26 & $M$ & $130 / 70$ & Indian & \\
\hline HUM-09 & 23 & $\mathrm{~F}$ & $115 / 80$ & Indian & $\begin{array}{c}\text { Irregular } \\
\text { cardiac cycle }\end{array}$ \\
\hline HUM-10 & 26 & $\mathrm{~F}$ & $120 / 80$ & Caucasian & \\
\hline
\end{tabular}

hertz, were measured and the following linear equation was the best fit with a correlation factor of 1.0:

$$
V_{0}(m V)=-2.2724+0.3455 f_{\text {in }}(\mathrm{Hz}) .
$$

The system output was monitored by a digital voltmeter (Fluke Model 45). The calibration was performed up to an input frequency of $24 \mathrm{kHz}$.

Through experimentation with the Tygon tube model shown in Figure 3, it was found that the optical signals corresponding to plot (b) of Figures 6 and 7 and $V_{0}$ in Figure 5 were well matched with the theoretical profiles of $v_{v}$, which were calculated with Eqs. (3) through (7) and given in plot (a) of Figures 6 and 7, when considering the shape and width, the number, and timing of each peak. Since the piezo sensor signal is also proportional to the time derivative of pressure profile, ${ }^{3}$ the piezo signal should be in phase with the optical signal and have both a positive and a negative pulse for each pressure pulse [see plot (c) of Figures 6 and 7].

Optical signals and reconstructed pressure profiles at various palpable sites of arterial pulsation are shown in Figure 8. The optical signals were taken from a single subject to allow appropriate comparison of the profiles. The arterial pressure profile was reconstructed from the optical signal, and is shown at the right. Because of compounding factors due to skin vibration, such as skin thickness, viscoelasticity, and optical property, the reconstructed pressure profiles are somewhat different from the empirically known standards, and the numerical values of the pressure profiles in Figure 8 are not given.

The signals detected superficial to the radial artery of normal and cardiac patients are shown in

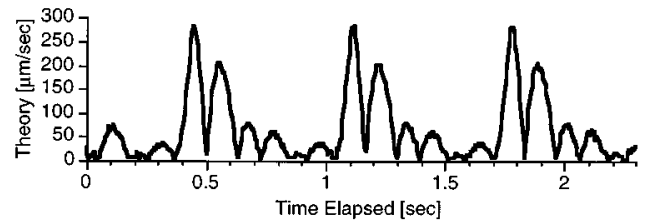

(a)

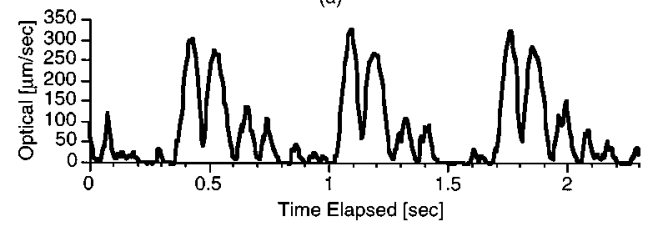

(b)

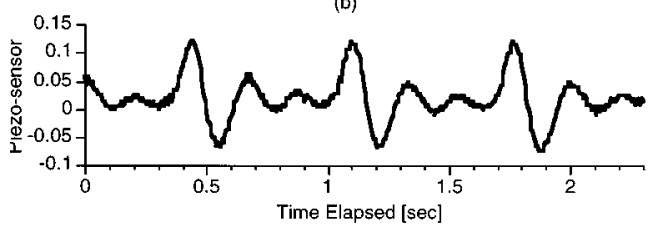

(c)

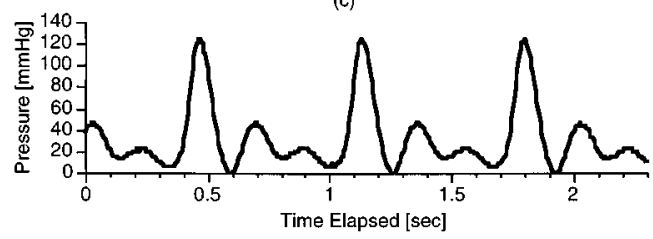

(d)

Fig. 6 Phantom test on the thick side of the gelatin layer. The signals were taken at side- $T$ indicated in Figure 3 . The unit for the piezo signal is arbitrary. Similarities of profiles are found between (a) and (b) plots for peaks, widths, duration, and timing of a pulse train. Plot $(c)$ is the signal obtained with a touch-type piezo sensor. The transmural pressure applied on the tube wall is shown as plot (d). The pressure wave form follows a pattern of postsurgical blood pressure at the radial artery. The pressure signal was measured with a physiological pressure transducer (Bell \& Howell, Model 4-327-0010). 


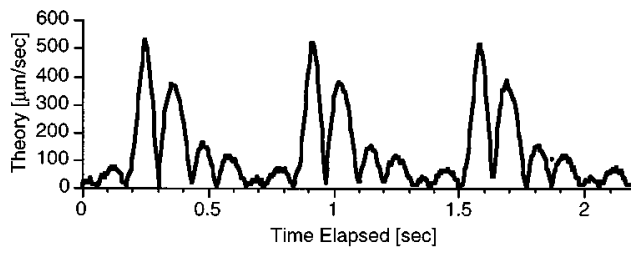

(a)

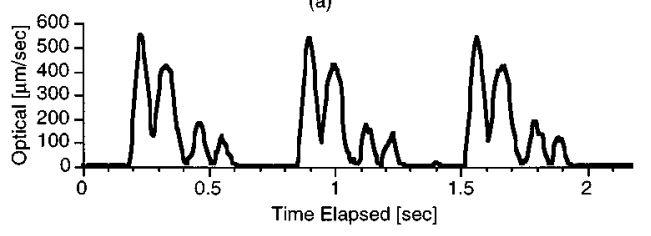

(b)

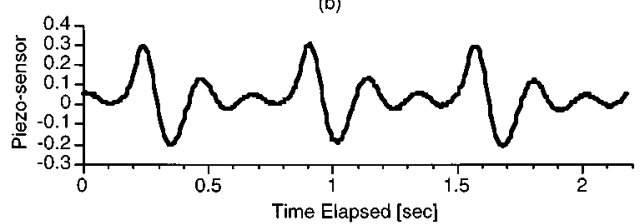

(c)

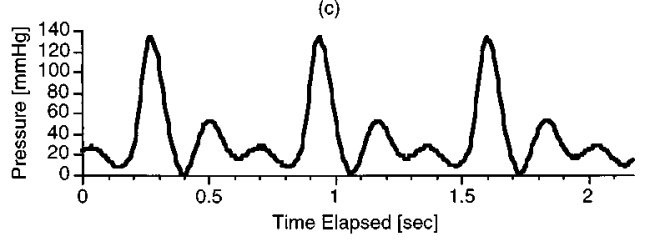

(d)

Fig. 7 Phantom test on the thin side of the gelatin layer: The signals were taken on side- $S$ indicated in Figure 3 . The peak vibration velocity is higher than that of the signal shown in Figure 6, which is a result of the reduced loading effects of the gelatin layer.

Figures 9(a) and 9(b). The amplitude and width of a primary pulse are found to be similar in both signals; however, it is the secondary pulse, representing the downstroke phase of blood pressure, that is not clearly seen in plot (b). Measurements were also performed on two other subjects superficial to the dorsalis pedis artery. The site of measurement was the dorsum of the foot lateral to the extensor tendon of the first toe. The results are shown in Figure 10. The secondary peak is closer to the primary peak and has a broader width, which can be a combination of several peaks. Therefore, the blood pressure profile is expected to be a rapid upstroke and slow downstroke in the diastolic phase. The theoretical profile of vessel wall motion shown in Figure 2(c) also shows a similar change.

Signals due to chest wall motion are shown in Figure 11. The chest wall vibrates at low frequencies, usually less than $20 \mathrm{~Hz}{ }^{13}$ The complex signal profile is due to the elastic wave that results from pericardial motion and cardiac acoustic waves. However, when the optical signal was taken at the mitral valve auscultation point, it was smaller in amplitude but wider in primary pulse width, which can be attributed to cardiac apex motion of low frequency and large amplitude vibration.

The results for the optical reflectivity of the skin surface are shown in Figure 12. Compared with the
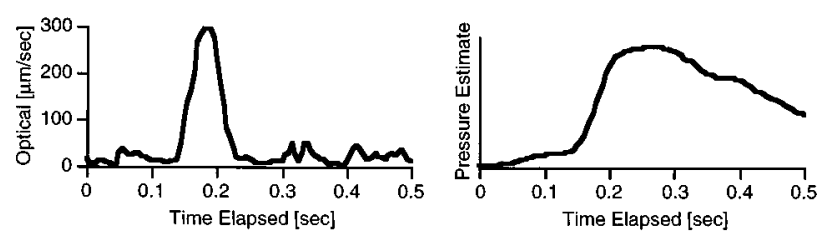

(a) Brachial Artery
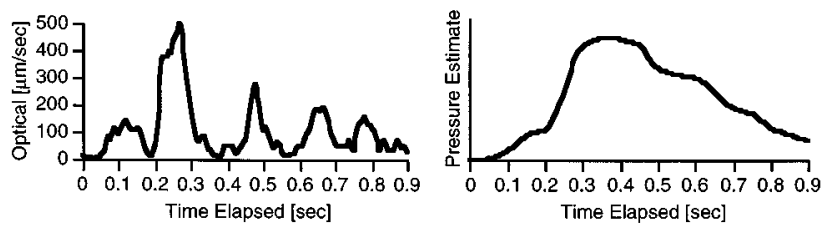

(b) Temporal Artery
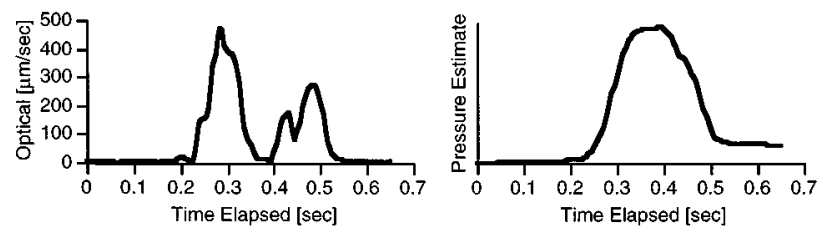

(c) Femoral Artery
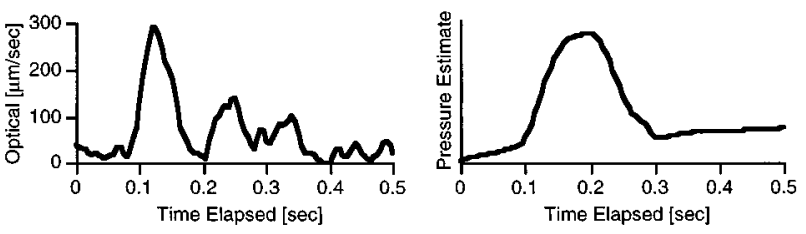

(d) Posterior Tibial Artery

Fig. 8 Optical signals measured at arterial palpation sites and reconstructed pressure profiles. The optical signals were taken from subject HUM-04. Only the major peaks in the optical signal are demonstrated, so the actual cardiac cycle lengths are longer than those shown. The plots shown at left correspond to the optical signals taken at various palpable sites of the arterial pulse from the same subject, and those shown at right correspond to the reconstructed pressure profiles obtained by running integration (rectangular window, $5 \mathrm{~ms}$ data interval) of optical signals, which is a procedure inverse to those used to obtain the vessel wall motion profiles in Figure 2.

control case shown as plot (a), the signal taken with a film shown as plot (b) shows improved signal-tonoise ratio (SNR) and appears to better define the secondary peaks, which are due to attenuation of skin vibration. The film was gently placed in apposition to the skin surface by a stethoscope bell (3M, Littman). A painted film contributed to enhanced laser reflection and worked as a mechanical highpass filter that suppressed low-frequency vibrations. Figure 12(d) shows the signal taken from the skin painted at the laser spot. Compared with (a), the SNR was significantly improved and the secondary and tertiary peaks were also enhanced. Increased optical reflectivity by skin painting could result in more optical power coupled into the fiber tip, thus improving the SNR of the Doppler signal. 


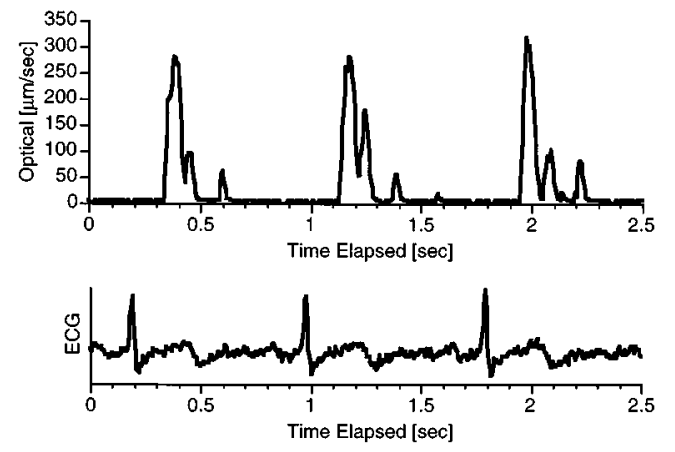

(a) HUM-01

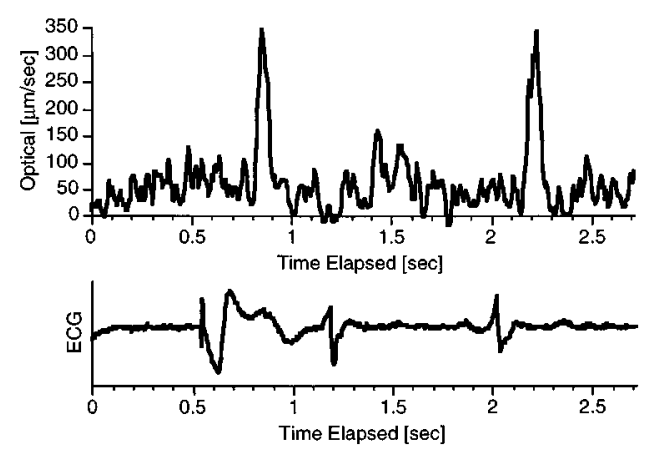

(b) HUM-05

Fig. 9 Optical signals for human subjects. These signals were obtained near the left radial arteries of subjects HUM-O 1 (normal) and HUM-05 (cardiac).

\section{DISCUSSION}

During the experiments, a subject sitting on a chair was exposed to ambient ground vibration. Signal artifacts related to global motion of a subject need to be minimized to improve sensitivity to the desired surface waves. As shown in Figure 4, we used a simple stethoscope bell-type adapter at the distal end of a light guide. The role of the adapter was to synchronize the mechanical motions of a light guide with global skin surface, which minimized the motion-related artifacts in a detected signal. Further research may be necessary to see whether this stretches or alters the skin at the sensing site, thus perturbing the natural skin surface vibration effect.

The minimum vibration velocity that the system could detect depended on the cutoff frequency of the low-pass filter (see Figure 5). Because of environmental noise and the subject's involuntary movement, the pass band of the Doppler signal was from $1.157 \mathrm{kHz}$ to $19.56 \mathrm{kHz}$, and the minimum velocity was $366.2 \mu \mathrm{m} / \mathrm{s}$. However, because the filters used in the system were not ideal, the actual pass band was wider. Therefore, noise due to the low-frequency motion of a subject, and environmental noise, such as building vibration, could not be completely removed and contributed signals around zero.

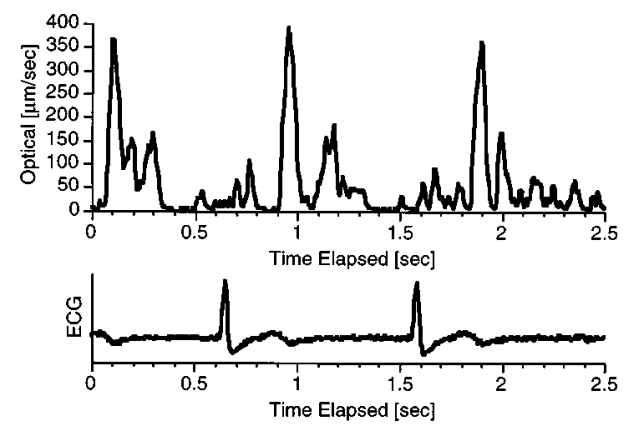

(a) HUM-08

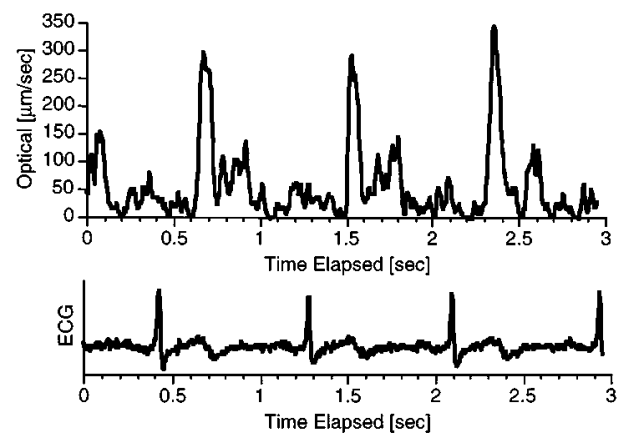

(b) HUM-04

Fig. 10 Optical signals for human subjects. These signals were obtained near the left dorsalis pedis artery of normal subjects HUM-08 and HUM-04.

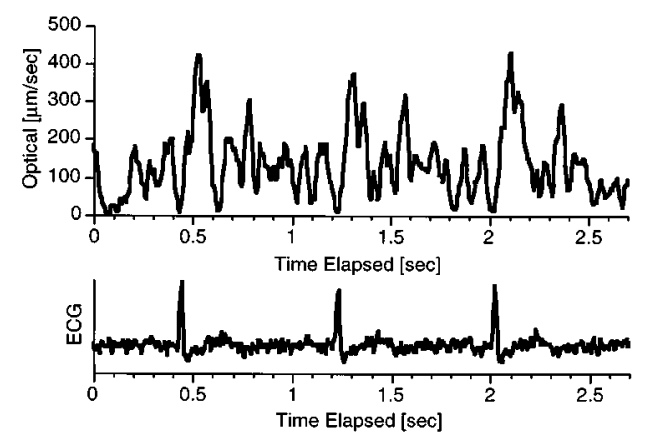

(a) Tricuspid valve points of chest auscultation

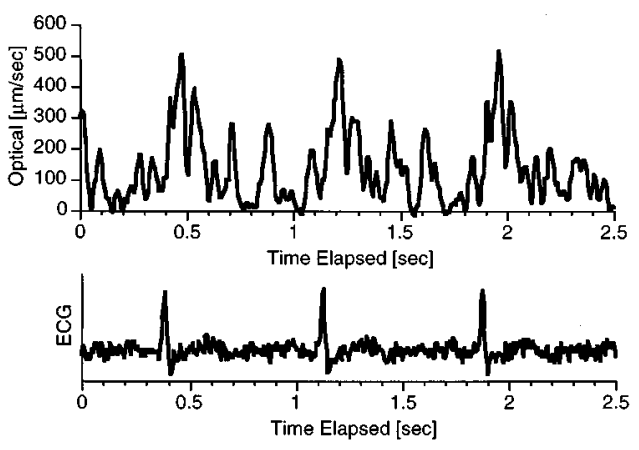

(b) Mitral valve points of chest auscultation

Fig. 11 Optical signals taken from the chest of human subjects. The signals were measured at the chest auscultation points for tricuspid and mitral valves of human subject HUM-04. 

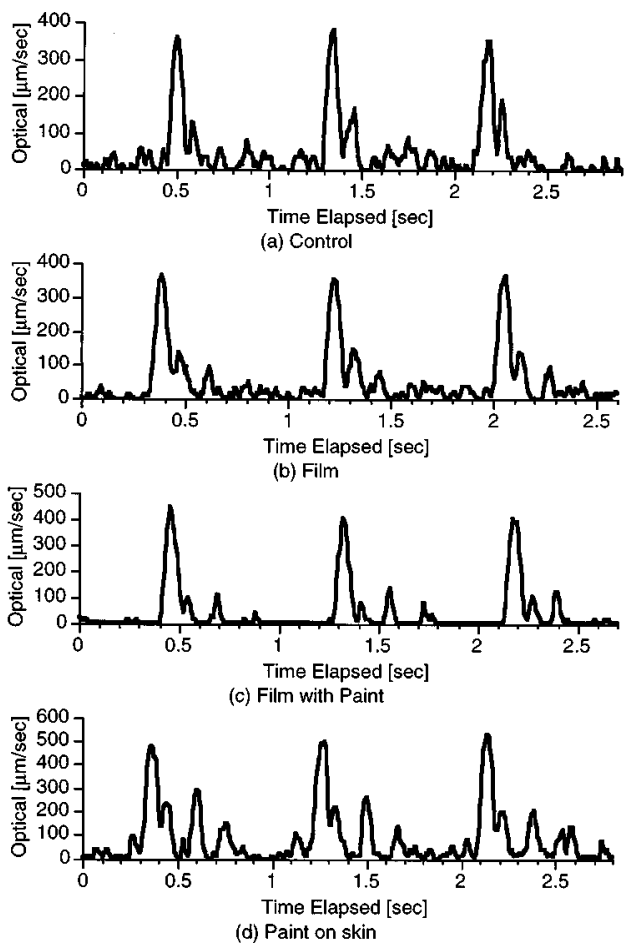

Fig. 12 Blocking of laser light penetration into skin layers. The signals shown were measured superficial to the left radial artery. Plot(a) is the control and plots (b), (c), and (d) correspond to three methods of controlling light penetration: (1) blocking light penetration into skin layers with optically opaque film, (2) blocking by painted film at the point of laser focus, and (3) blocking by direct painting on the skin surface.

In the instrumentation shown in Figure 5, the frequency to voltage converter was not designed to discriminate signals according to the direction of skin surface movement. Therefore, there were no quadrature components in the optical signals, while the compressive and stretching forces could be discriminated in the piezo signal. To obtain the direction-dependent signals from the present system, modulating the probing ray with either an acousto-optic or an electro-optics modulator and a quarter wave retarder, which is used in ultrasound imaging, can be tried.

As the results have shown, the vessel wall motion profiles followed the characteristics of a blood pressure wave; the amplitude of the major peak related to the slope of the first upstroke, the pulse coupling interval of the major peaks related to the width of the major pressure wave, and the number of peaks was related to the prominence and depression of the blood pressure pulse train. The number of peaks in the vibration signal was directly related to the number of pulses, since one pressure pulse corresponds to two pulses in the optical signal.

At any one pulsation site, signal morphology varied for different subjects because of dermal structure, cardiac function, and the thickness of skin layers. The skin layers determine the photon scattering/absorption and mechanical damping or delaying effects on the vessel wall motion.,19 Furthermore, the input signal to the frequency-tovoltage converter consisted of both the pure Doppler component and the threshold signal, which was adjusted according to the degree of environmental noise and involuntary motion of the subject. In performing experiments, the threshold level was appropriately adjusted for each subject.

Even though the experimental system was initially developed as laboratory-based instrumentation to measure pulsation, the system could be used clinically for epidemiological study of the aging of the cardiovascular system, since the instrumentation is low cost and noninvasive. For clinical application, the performance criteria of the system, such as specificity and sensitivity, should be evaluated to compare the signals before and after intentional alteration of pulse contours, such as the Valsalva maneuver, exercise, or even with daily activity. The results also suggest that signal patterns did not appear to change significantly with melanin concentration in the dermal layer or with the race of a subject.

\section{CONCLUSION}

Present research, which allows arterial wall motion to be measured in the extremities, should have a greater ability to assess the underlying viability of the vascular system. It was demonstrated that the measurement system developed responded to skin vibration velocity, which was proportional to the time derivative of the blood pressure profile within the underlying vasculature. While more work is necessary to examine the clinical implications of the results obtained, it is clear that optical means can be used to investigate surface vibrations induced by underlying arterial wall motion and, even with its existing limitations, the present system can have the potential to assess the propagation velocity of a pulse wave along multiple segments of an arterial tree.

\section{REFERENCES}

1. B. B. Bates, A guide to Physical Examination, 5th ed., Chap. 9, Lippincott, Philadelphia, PA (1991).

2. W. W. Nichols and M. F. O'Rourke, McDonald's Blood Flow in Arteries: Theoretical, Experimental and Clinical Principles, 3rd ed., Lea \& Febiger, Philadelphia, PA (1990).

3. M. D. Stern, "In vivo evaluation of microcirculation by coherent light scattering," Nature 254, 56-58 (1975).

4. M. D. Stern, "Laser Doppler velocimetry in blood and multiply scattering fluids: theory," Appl. Opt. 24(13), 1968-1986 (1985).

5. R. Bonner and R. Nossal, "Method for laser Doppler measurement of blood flow in tissue," Appl. Opt. 20(2), 20972107 (1981).

6. D. Watkins and A. Halloway, "An instrument to measure cutaneous blood flow using the Doppler shift of laser light," IEEE Trans. Biomed. Eng. BME-25(1), 28-33 (1976).

7. T. Eiju, M. Nagai, and K. Matsuda, "Microscopic laser Doppler velocimeter for blood velocity measurement," Opt. Eng. 32, 15-20 (1993).

8. K. Wardell, A. Jakobsson, and G. E. Nillson, "Laser Doppler 
perfusion imaging by dynamic light scattering," IEEE Trans. Biomed. Eng. 40(4), 309-316 (1993).

9. J. S. Lee, "On the coupling and detection of motion between an artery with a localized lesion and its surrounding tissue," J. Biomech. 7, 403-409 (1974).

10. J. R. Womersley, "Oscillating flow in arteries: the constrained elastic tube as a model of arterial flow and pulse transmission," Phys. Med. Biol. 2, 175-187 (1957).

11. J. C. Bramwell and A. V. Hill, "The velocity of the pulse wave in man," Proc. R. Socl. (Lond.) 93, 298-306 (1922).

12. D. H. Bergel, "The static elastic properties of the arterial wall," J. Physiol. 56, 445-457 (1961).

13. H. D. Hong, "Optical interferometric measurement of skin vibration for the diagnosis of cardiovascular diseases," Ph.D. Diss., University of Connecticut, Storrs, CT (1994).

14. M. F. O'Rourke, R. Kelly, and A. Avolio, The Arterial Pulse, Lea \& Febiger, Philadelphia, PA (1992).
15. W. H. Hoppman and L. Wan, "Large deformation of elastic tubes," J. Biomech. 3, 593-600 (1970).

16. D. F. Young and F. Tsai, "Flow characteristics in models of arterial stenosis, II. Unsteady flow," J. Biomech. 6, 547-559 (1973).

17. J. K. Raines, M. Y. Jaffrin, and A. H. Shaprio, "A computer simulation of arterial dynamics in the human leg," J. Biomech. 7, 77-91 (1974).

18. M. Mirdha, S. Odman, and P. A. Oberg, "Mechanical pulse wave propagation in gel: normal and oedematous tissues," J. Biomech. 25(12), 1213-1218 (1992).

19. R. R. Anderson and J. A. Parrish, "Optical properties of human skin," in J. D. Regan and J. A. Parrish, eds., The Science of Photomedicine, Chap. 6, Plenum Press, New York (1982). 